\title{
Low Levels of Polymorphisms and Negative Selection in Plasmodum knowlesi Merozoite Surface Protein 8 in Malaysian Isolates
}

\author{
Md Atique Ahmed', Hae-Ji Kang², Fu-Shi Quan ${ }^{1,3 *}$ \\ ${ }^{1}$ Department of Medical Zoology, School of Medicine, Kyung Hee University, Seoul 02447, Korea; '2Department of Biomedical Science, Graduate \\ School, Kyung Hee University, Seoul 02447, Korea; ${ }^{3}$ Medical Research Center for Bioreaction to Reactive Oxygen Species and Biomedical Science \\ Institute, School of Medicine, Graduate School, Kyung Hee University, Seoul 02447, Korea
}

\begin{abstract}
Human infections due to the monkey malaria parasite Plasmodium knowlesi is increasingly being reported from most Southeast Asian countries specifically Malaysia. The parasite causes severe and fatal malaria thus there is a need for urgent measures for its control. In this study, the level of polymorphisms, haplotypes and natural selection of full-length pkmsp8 in 37 clinical samples from Malaysian Borneo along with 6 lab-adapted strains were investigated. Low levels of polymorphism were observed across the full-length gene, the double epidermal growth factor (EGF) domains were mostly conserved, and non-synonymous substitutions were absent. Evidence of strong negative selection pressure in the non-EGF regions were found indicating functional constrains acting at different domains. Phylogenetic haplotype network analysis identified shared haplotypes and indicated geographical clustering of samples originating from Peninsular Malaysia and Malaysian Borneo. This is the first study to genetically characterize the full-length $m s p 8$ gene from clinical isolates of $P$. knowlesi from Malaysia; however, further functional characterization would be useful for future rational vaccine design.
\end{abstract}

Key words: Plasmodium knowlesi, pkmsp8, merozoite surface protein 8, genetic diversity, natural selection, haplotype, polymorphism, Malaysia

Plasmodium knowlesi, a zoonotic malaria parasite is now considered as the fifth Plasmodium species infecting humans as large number of cases have been reported from Southeast Asian countries, specifically Malaysia [1,2]. Within Malaysia, the highest number of human infections have been reported from Malaysian Borneo since 2004 [3-5] and very recently from Peninsular Malaysia too [6] highlighting the need of effective control measures and also the development of effective vaccines. With its 24-hr erythrocytic cycle, rapid increase in parasite counts has been shown to be associated with severe malaria and sometimes fatal $[7,8]$. Almost $70-78 \%$ of malaria cases reported from Malaysian Borneo (Sarawak and Kudat, Sabah) were due to $P$. knowlesi $[5,9]$. Recent genomic studies on $P$. knowlesi in clinical isolates of Malaysian Borneo have identified at least 3 sub-populations, which are highly diverse, 2 of the populations were associated with primary primate hosts and

\footnotetext{
- Received 17 February 2019, revised 15 July 2019, accepted 17 July 2019.

*Corresponding author (fquan01@gmail.com)

(C) 2019, Korean Society for Parasitology and Tropical Medicine

This is an Open Access article distributed under the terms of the Creative Commons

Attribution Non-Commercial License (http://creativecommons.org/licenses/by-nc/4.0)

which permits unrestricted non-commercial use, distribution, and reproduction in any

medium, provided the original work is properly cited.
}

one with geographical location $[10,11]$. Analysis with mitochondrial genes in P. knowlesi clinical isolates and macaques also identified 2 distinct clusters which clustered geographically to Malaysian mainland and Malaysian Borneo [12].

Vaccine design and vaccine efficacy studies require the understanding of the extent and dynamics of genetic diversity in target antigens from malaria endemic regions. Major vaccine candidates studied in P. falciparum (like CSP, AMA1) show high genetic diversity and evolve under positive natural selection in the field in order to evade host immune pressure and thus are excellent targets for protective immunity but high variability also leads to non-efficacious vaccine trial due to strain-specific immune response [13]. For decades, vaccine research against malaria has primarily focused on $P$. falciparum and $P$. vivax and until date, not a single efficacious vaccine has been found which provides $100 \%$ protection. Merozoite surface proteins (MSPs) are recognized as potential vaccine candidates as they have been found to elicit a strong antibody response in patients and some molecules have shown strong inhibitory activity in RBCs in-vitro [14-16]. However, high antigenic variations within parasite populations are considered as one of the major hurdles in developing an efficacious and 
strain-transcending vaccine. Extensive genetic diversity has been observed in clinical isolates of $P$. knowlesi both in the genetic level as well as in the genomic level and several known ortholog vaccine antigens have shown similar levels of high diversity $[10,11,17-20]$. These studies highlight the complexities involved in $P$. knowlesi vaccine design thus a rational approach would be necessary. Thus, it is important to identify potential blood stage parasite antigens, which are essential for its survival, low in polymorphism in the endemic region and show significant immune response in patient serum. Merozoite surface protein 8 (MSP8) contains 2 copies of a conserved epidermal growth factor (EGF)-like domain at the carboxyl terminal that is anchored to the membrane via glycosylphosphatidylinositol (GPI) membrane anchor [17]. Specific binding of MSP8-peptides to human RBCs have been reported in $P$. falciparum suggesting an essential role in parasite invasion [21] and naturally acquired humoral and cell mediated immune response in patient sera have been observed in $P$. vivax [22]. Genetic studies on MSP8 of $P$. falciparum and P. vivax from world-wide isolates (from different geographical locations) indicated that the gene is under purifying selection and has low levels of polymorphism [23]. However, no studies have been conducted in $P k m s p 8$ from clinical samples. Thus, this study was designed to determine the level of diversity, haplotypes and natural selection acting at the full-length gene and its domains from clinical isolates from Sarawak, Malaysian Borneo.

Pkmsp8 sequences were downloaded for 37 clinical isolates originating from Kapit, Betong and Sarikei from Sarawak, Malaysian Borneo along with 6 long-time isolated lines originated from Mainland Malaysia along with the H-strain ( $\mathrm{PKNH}_{-}$ 1031500) [11]. The sequence data with accession numbers are same as used for a previous study [17]. The PkMSP8 domains were characterized based on the published ortholog in PvMSP8 (PVX_097625) [22]. Sequence diversity $(\pi)$, the number of polymorphic sites, number of synonymous and non-synonymous substitutions, haplotype diversity (Hd) and number of haplotypes $(\mathrm{H})$ within the pkmsp8 sequences was determined by DnaSP v5.10 software [24]. Natural selection was determined at the intra-population level by calculating the rates of synonymous substitutions per synonymous site (dS) and nonsynonymous substitutions per non-synonymous site $(\mathrm{dN})$ were computed by using Nei and Gojobori's method and robustness were estimated by the bootstrap method with 1000 pseudo-replicates as implemented in the MEGA 5.0 software [25]. If dN-dS differences were positive, it corresponds to posi- tive natural selection and negative values corresponds to negative selection. Also, codon based Z-test for selection was implemented with MEGA software to test the significance using 1000 bootstrap values. Tajima's D, Fu \& Li's D* and F* tests were implemented in DnaSP v5.10 software. When Tajima's D, Fu \& Li's D* and $\mathrm{F}^{*}$ values are positive and significant, it indicates positive/balancing selection, whereas negative values suggest negative selection or population expansion. To test whether the pkmsp8 gene is under the influence of natural selection in the inter-species level, the robust McDonald and Kreitman (MK) test were performed with both P. coatneyi (PCOAH_00031550) and P. cynomolgi (PCYB_104050) msp8 gene as an out-groups using DnaSP v5.10 software [25]. Genealogical relationships were constructed between the pkmsp 8 haplotypes using the median-joining method in NETWORK software (version 4.6.1.2, Fluxus Technology Ltd., Suffolk, UK).

The schematic structure of $p k m s p 8$ gene and its domains were demarcated based on the P. vivax MSP8 protein (Fig. 1A). There was an asparagine-rich region (ASN) upstream the double EGF

A

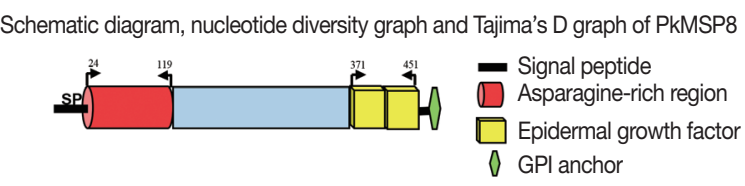

B

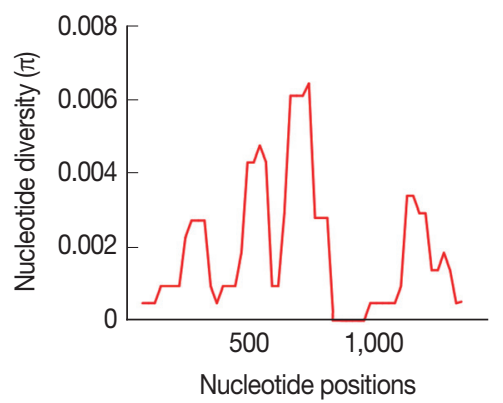

C

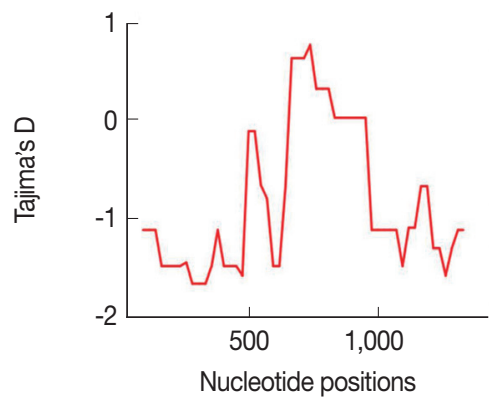

Fig. 1. (A) Schematic diagram of Plasmodium knowlesi MSP8 domains (B) Graphical representation of nucleotide diversity $(\pi)$ within 37 full-length pkmsp8 genes ( $n=1,431$ bp) from Malaysian Borneo and the lab-adapted strains. The PKMSP8 domains are marked above. (C) Graphical representation of Tajima's D value across the MSP8 gene. 
domains, which was similar to P. vivax MSP8 [22] (Fig. 1A). Within the full-length $p k m s p 8$ sequences $(\mathrm{n}=43,1,431 \mathrm{bp})$, there were 24 polymorphic sites (1.67\%) leading 17 synonymous and 7 non-synonymous substitutions. We found 8 parsimony informative sites and 16 singleton variable sites. The overall nucleotide diversity was lower $(\pi=0.0018 \pm$ SD 0.00028$)$ compared to its ortholog in P. vivax and P. falciparum which is relatively conserved (Table 1) [23]. Low levels of polymorphism were observed across the full-length $p k m s p 8$ gene and the majority of the SNPs were synonymous substitutions. Similar reports of high number of synonymous substitutions in $m s p 1 p$ and $m s p 1$ genes were observed in P. knowlesi clinical isolates with negative/purifying natural selection $[20,26]$. The diversity towards the non-EGF and the double EGF domains were of similar levels ( $\pi=0.00010-0.00016)$, however, the number of SNPs towards the non-EGF domains was higher than EGF domain (Table 1) indicating the EGF domains were conserved. Sequence alignment showed that non-synonymous substitutions were absent within the C-terminal double EGF domains compared with the non-EGF domain (Table 1). It is to be noted that the singleton sites were scattered more towards the non-EGF domain ( $\mathrm{Si}=13)$, while the EGF domains had only three. The sliding window plot analysis (window length 100 bp and step size $25 \mathrm{bp}$ ) also revealed that the overall diver- sity ranged from 0 to 0.0061 and the C-terminal double EGF domains containing the $19 \mathrm{kDa}$ domain showed lower diversity (Fig. 1B). Due to the high number of singletons in the nonEGF domains, the number of haplotypes and haplotype diversities were higher compared to the EGF domain within the pkmsp8 gene (Table 1). The 12-cysteine residues within the $2 \mathrm{EGF}$ domains at the $19 \mathrm{kDa}$ domain were conserved within the clinical isolates indicating functional conservation.

To determine whether natural selection contributes to the polymorphism in the pkmsp8 full-length gene as well as at each domain (EGF and non-EGF), multiple tests were conducted; at the intra-species level significant negative value was observed at the full-length gene and at the non-EGF domain (Table 1) indicating $\mathrm{dN}<\mathrm{dS}$. Additional statistical test for neutrality; Tajimas' $\mathrm{D}$, Li and Fu's $\mathrm{F}^{*}$ and $\mathrm{D}^{*}$ statistics also showed significant negative values for the full-length gene and the non-EGF domain indicating negative/purifying natural selection and parasite population expansion in Malaysian Borneo. However, it was observed that even though test results were with negative values for the double EGF domain, it was not statistically significant and the absence of non-synonymous substitutions within the double EGF domain indicate an absence of natural selection within the domain and functional conservation. Interestingly, all the 12 cysteine residues within the double EGF domains were conserved

Table 1. Estimates of nucleotide diversity, natural selection, haplotype diversity and neutrality indices of pkmsp8 and its domains within 43 isolates

\begin{tabular}{|c|c|c|c|c|c|c|c|c|c|c|c|c|}
\hline \multirow{2}{*}{ Region } & \multirow{2}{*}{ SNPS } & \multirow{2}{*}{$\mathrm{Si}$} & \multirow{2}{*}{ Syn } & \multirow{2}{*}{ Non-syn } & \multirow{2}{*}{$\begin{array}{c}\text { No. } \\
\text { haplotype }\end{array}$} & \multicolumn{2}{|c|}{ Diversity $\pm S D$} & \multirow{2}{*}{$d_{N}-d_{S}$} & \multirow{2}{*}{$\begin{array}{c}\text { Codon } \\
\text { based z-test }\end{array}$} & \multirow{2}{*}{ Taj D } & \multirow{2}{*}{$\begin{array}{l}\text { Fu \& } \\
\text { Li's D }\end{array}$} & \multirow{2}{*}{$\begin{array}{l}\text { Fu \& } \\
\text { Li's F }\end{array}$} \\
\hline & & & & & & Haplotype & Nucleotide & & & & & \\
\hline Full-length & 24 & 16 & 17 & 7 & 18 & $0.776 \pm 0.004$ & $0.001 \pm 0.000$ & -2.5 & $P<0.05$ & -1.8 & & $-3.18^{a}$ \\
\hline Non-EGF domain & 19 & 13 & 11 & 7 & 15 & $0.707 \pm 0.075$ & $0.001 \pm 0.000$ & -1.9 & $P<0.05$ & -1.7 & $-3.12^{\mathrm{a}}$ & $-3.13^{\mathrm{a}}$ \\
\hline EGF domain & 5 & 3 & 5 & 0 & 6 & $0.371 \pm 0.091$ & $0.0016 \pm 0.000$ & -1.7 & $P>0.05$ & -1.4 & -1.7 & -1.9 \\
\hline
\end{tabular}

SNPs, single nucleotide polymorphisms; Si, singleton sites; Syn, Synonymous substitutions; Non-syn, Nonsynonymous substitutions; SD, Standard deviation.

${ }^{\mathrm{a}} \mathrm{P}<0.05$.

Table 2. McDonald-Kreitman tests on MSP8 of Plasmodium knowlesi and its domains with P. coatneyi and P. cynomolgi orthologs as outgroup sequences

\begin{tabular}{|c|c|c|c|c|c|c|c|c|}
\hline \multirow{3}{*}{ MSP8 } & \multicolumn{2}{|c|}{$\begin{array}{l}\text { Polymorphic change within } \\
\text { P. knowlesi }\end{array}$} & \multicolumn{4}{|c|}{ Fixed differences between species } & \multicolumn{2}{|c|}{ Neutrality index } \\
\hline & \multirow{2}{*}{ Syn } & \multirow{2}{*}{ NonSyn } & \multicolumn{2}{|c|}{ Pk vs Pco } & \multicolumn{2}{|c|}{$P k$ vs Pcy } & \multirow{2}{*}{$P k$ vs Pco } & \multirow{2}{*}{ Pk vs Pcy } \\
\hline & & & Syn & NonSyn & Syn & NonSyn & & \\
\hline Full-length & 17 & 7 & 52 & 58 & 52 & 68 & 0.41 & $0.33^{\mathrm{a}}$ \\
\hline Non-EGF domain & 10 & 4 & 45 & 57 & 42 & 66 & 0.31 & $0.23^{\mathrm{a}}$ \\
\hline EGF-domain & 4 & 0 & 9 & 4 & 13 & 4 & 0.00 & 0.00 \\
\hline
\end{tabular}

Syn, Synonymous sites; NonSyn, Non synonymous sites; Pk, Plasmodium knowlesi; Pcy, Plasmodium cynomolgi; Pco, Plasmodium coatneyi. 'Fisher's exact test; $P<0.05$. 


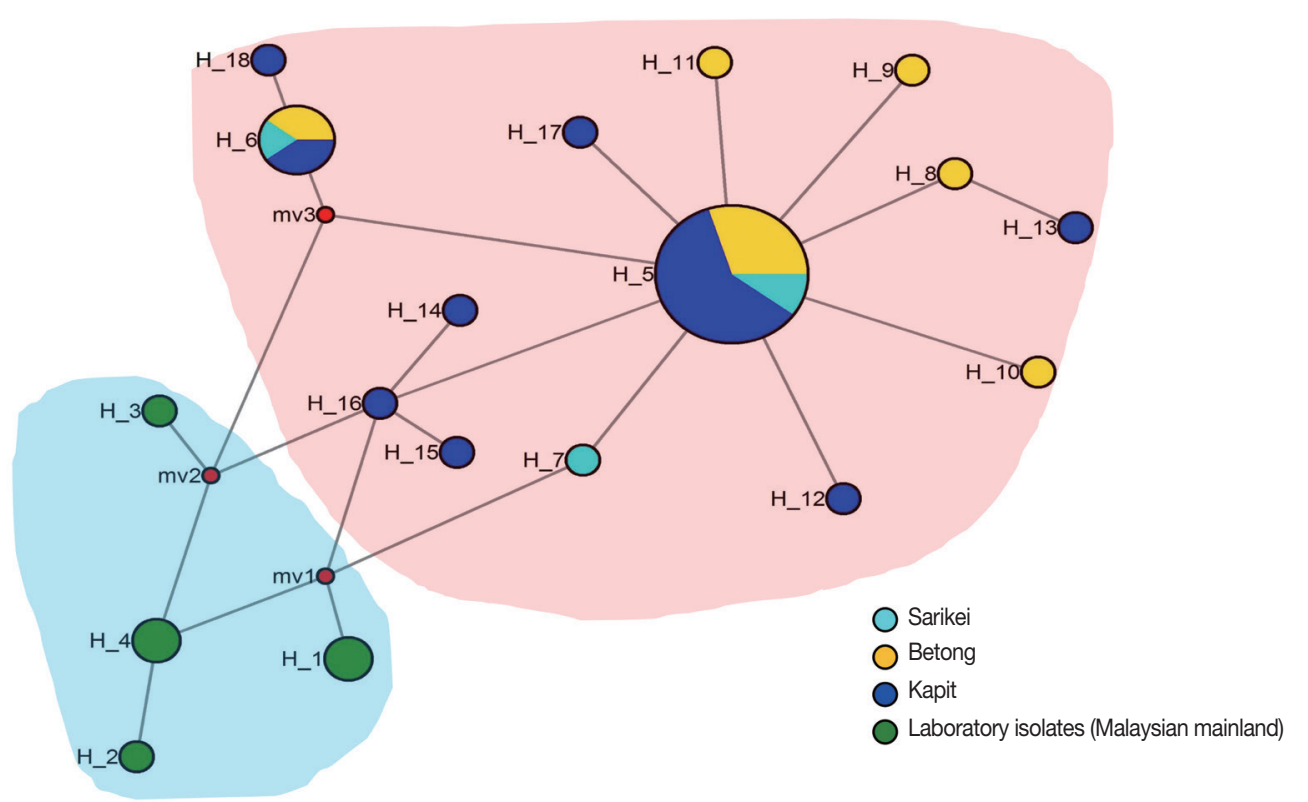

Fig. 2. Median-joining networks of Plasmodium knowlesi MSP8 haplotypes from Malaysia. The genealogical haplotype network shows the relationships among the 18 haplotypes present in the 43 sequences obtained from clinical isolates and lab adapted strains from 5 geographical regions of Malaysia. Each distinct haplotype has been designated a number $\left(H \_n\right)$. Circle sizes represent the frequencies of the corresponding haplotype (the number is indicated for those that were observed $>1 \times$ ). Distances between nodes are arbitrary. The small red circles are median vectors randomly generated by the software while constructing the network.

within the 43 isolates (including the lab adapted strains) indicating conserved functional activity. This conservation of the 12 cysteine residues was also observed for the C-terminal $19 \mathrm{kDa}$ fragment of PvMSP1P and PkMSP1P [26] and binding activity to reticulocytes in P. vivax have been reported [14,27-29]. Sliding window plot analysis of Tajima's D across the full-length pkmsp8 gene also indicated most values below 0 indicating purifying selection (Fig. 1C). Natural selection test using the MK test (at the inter-species level) with P. coatneyi and P. cynomolgi as orthologous sequences also showed similar results with strong negative/ purifying selection acting at the full-length and the non-EGF domain probably due to functional constraints and the domain may not be exposed to host immune pressure (Table 2).

Two distinct population clusters were observed; one originating from Malaysian mainland where the lab adapted strains originated (i.e., H, Malayan, Nuri, Hackeri, Philippine and $\mathrm{MR} 4 \mathrm{H}$ ) and the other sub-cluster was the clinical isolates from Sarawak, Malaysian Borneo (Fig. 2). Two major shared haplotypes were found between parasite populations from Kapit, Betong and Sarikei (H_5 and H_6) (Fig. 2) and these 2 haplotype clusters may represent the two distinct clusters observed from Malaysian Borneo. Similar findings with clinical isolates from Malaysian Borneo have been reported earlier with merozoite surface proteins $[17,20,26,30]$. It is interesting to note that MSP8 gene has very low levels of polymorphism compared to its orthologs in P. vivax and P. falciparum [23] and thus might be an ideal candidate for vaccine design against $P$. knowlesi. This may be due to the absence of host immune pressure in the EGF domains which is critical for binding to host cells. However, further studies characterizing the immunological and functional validation of the candidate would be necessary.

\section{ACKNOWLEDGMENT}

This work was supported by grants from the National Research Foundation of Korea (NRF) (2018R1A2B6003535, 2018R1A6A1A03025124).

\section{CONFLICT OF INTEREST}

The authors declare that they have no competing interests.

\section{REFERENCES}

1. Ahmed MA, Cox-Singh J. Plasmodium knowlesi - an emerging pathogen. ISBT Sci Ser 2015; 10: 134-140. 
2. Amir A, Cheong FW, de Silva JR, Liew JWK, Lau YL. Plasmodium knowlesi malaria: current research perspectives. Infect Drug Resist 2018; 11: 1145-1155.

3. Singh B, Kim Sung L, Matusop A, Radhakrishnan A, Shamsul SS, Cox-Singh J, Thomas A, Conway DJ. A large focus of naturally acquired Plasmodium knowlesi infections in human beings. Lancet 2004; 363: 1017-1024.

4. Barber BE, William T, Grigg MJ, Menon J, Auburn S, Marfurt J, Anstey NM, Yeo TW. A prospective comparative study of knowlesi, falciparum, and vivax malaria in Sabah, Malaysia: high proportion with severe disease from Plasmodium knowlesi and Plasmodium vivax but no mortality with early referral and artesunate therapy. Clin Infect Dis 2013; 56: 383-397.

5. Barber BE, William T, Jikal M, Jilip J, Dhararaj P, Menon J, Yeo TW, Anstey NM: Plasmodium knowlesi malaria in children. Emerg Infect Dis 2011; 17: 814-820.

6. Yusof R, Lau YL, Mahmud R, Fong MY, Jelip J, Ngian HU, Mustakim S, Hussin HM, Marzuki N, Mohd Ali M. High proportion of knowlesi malaria in recent malaria cases in Malaysia. Malar J 2014; $13: 168$.

7. William T, Menon J, Rajahram G, Chan L, Ma G, Donaldson S, Khoo S, Frederick C, Jelip J, Anstey NM, Yeo TW. Severe Plasmodium knowlesi malaria in a tertiary care hospital, Sabah, Malaysia. Emerg Infect Dis 2011; 17: 1248-1255.

8. Willmann M, Ahmed A, Siner A, Wong IT, Woon LC, Singh B, Krishna S, Cox-Singh J. Laboratory markers of disease severity in Plasmodium knowlesi infection: a case control study. Malar J 2012; 11: 363.

9. Daneshvar C, Davis TM, Cox-Singh J, Rafa'ee MZ, Zakaria SK, Divis PC, Singh B. Clinical and laboratory features of human Plasmodium knowlesi infection. Clin Infect Dis 2009; 49: 852-860.

10. Pinheiro MM, Ahmed MA, Millar SB, Sanderson T, Otto TD, Lu WC, Krishna S, Rayner JC, Cox-Singh J. Plasmodium knowlesi genome sequences from clinical isolates reveal extensive genomic dimorphism. PLoS One 2015; 10: e0121303.

11. Assefa S, Lim C, Preston MD, Duffy CW, Nair MB, Adroub SA, Kadir KA, Goldberg JM, Neafsey DE, Divis P, Clark TG, Duraisingh MT, Conway DJ, Pain A, Singh B. Population genomic structure and adaptation in the zoonotic malaria parasite Plasmodium knowlesi. Proc Natl Acad Sci USA 2015; 112: 1302713032.

12. Yusof R, Ahmed MA, Jelip J, Ngian HU, Mustakim S, Hussin HM, Fong MY, Mahmud R, Sitam FA, Japning JR, Snounou G, Escalante AA, Lau YL. Phylogeographic evidence for 2 genetically distinct zoonotic Plasmodium knowlesi parasites, Malaysia. Emerg Infect Dis 2016; 22: 1371-1380.

13. Takala SL, Coulibaly D, Thera MA, Batchelor AH, Cummings MP, Escalante AA, Ouattara A, Traoré K, Niangaly A, Djimdé AA, Doumbo OK, Plowe CV. Extreme polymorphism in a vaccine antigen and risk of clinical malaria: implications for vaccine development. Sci Transl Med 2009; 1: 2ra5.

14. Cheng Y, Shin EH, Lu F, Wang B, Choe J, Tsuboi T, Han ET. Antigenicity studies in humans and immunogenicity studies in mice: an MSP1P subdomain as a candidate for malaria vaccine development. Microbes Infect 2014; 16: 419-428.

15. Valderrama-Aguirre A, Quintero G, Gómez A, Castellanos A, Pérez Y, Méndez F, Arévalo-Herrera M, Herrera S. Antigenicity, immunogenicity, and protective efficacy of Plasmodium vivax MSP1 PV200l: a potential malaria vaccine subunit. Am J Trop Med Hyg 2005; 73: 16-24.

16. O'Donnell RA, Saul A, Cowman AF, Crabb BS: Functional conservation of the malaria vaccine antigen MSP-119across distantly related Plasmodium species. Nat Med 2000; 6: 91-95.

17. Ahmed MA, Quan FS. Plasmodium knowlesi clinical isolates from Malaysia show extensive diversity and strong differential selection pressure at the merozoite surface protein 7D (MSP7D). Malar J 2019: 18: 150.

18. Ahmed MA, Fong MY, Lau YL, Yusof R. Clustering and genetic differentiation of the normocyte binding protein (nbpxa) of Plasmodium knowlesi clinical isolates from Peninsular Malaysia and Malaysia Borneo. Malar J 2016; 15: 241.

19. Ahmed AM, Pinheiro MM, Divis PC, Siner A, Zainudin R, Wong IT, Lu CW, Singh-Khaira SK, Millar SB, Lynch S, Willmann M, Singh B, Krishna S, Cox-Singh J Disease progression in Plasmodium knowlesi malaria is linked to variation in invasion gene family members. PLoS Negl Trop Dis 2014; 8: e3086.

20. Ahmed MA, Chu KB, Vythilingam I, Quan FS. Within-population genetic diversity and population structure of Plasmodium knowlesi merozoite surface protein 1 gene from geographically distinct regions of Malaysia and Thailand. Malar J 2018; 17: 442.

21. Puentes A, García J, Ocampo M, Rodríguez L, Vera R, Curtidor H, López R, Suarez J, Valbuena J, Vanegas M, Guzman F, Tovar D, Patarroyo ME. P. falciparum: merozoite surface protein- 8 peptides bind specifically to human erythrocytes. Peptides 2003; 24 : 1015-1023.

22. Cheng Y, Wang B, Changrob S, Han JH, Sattabongkot J, Ha KS, Chootong P, Lu F, Cao J, Nyunt MH, Park WS, Hong SH, Lim CS, Tsuboi T, Han ET. Naturally acquired humoral and cellular immune responses to Plasmodium vivax merozoite surface protein 8 in patients with P. vivax infection. Malar J 2017; 16: 211.

23. Pacheco MA, Elango AP, Rahman AA, Fisher D, Collins WE, Barnwell JW, Escalante AA. Evidence of purifying selection on merozoite surface protein 8 (MSP8) and 10 (MSP10) in Plasmodium spp. Infect Genet Evol 2012; 12: 978-986.

24. Librado P, Rozas J. DnaSP v5: a software for comprehensive analysis of DNA polymorphism data. Bioinformatics 2009; 25: 1451-1452.

25. Tamura K, Peterson D, Peterson N, Stecher G, Nei M, Kumar S. MEGA5: molecular evolutionary genetics analysis using maximum likelihood, evolutionary distance, and maximum parsimony methods. Mol Biol Evol 2011; 28: 2731-2739.

26. Ahmed MA, Fauzi M, Han ET. Genetic diversity and natural selection of Plasmodium knowlesi merozoite surface protein 1 paralog gene in Malaysia. Malar J 2018; 17: 115.

27. Han JH, Cho JS, Cheng Y, Muh F, Yoo WG, Russell B, Nosten F, Na S, Ha KS, Park WS, Hong SH, Han ET. Plasmodium vivax mer- 
ozoite durface protein 1 paralog as a mediator of parasite adherence to reticulocytes. Infect Immun 2018; 86.

28. Min HMK, Changrob S, Soe PT, Han JH, Muh F, Lee SK, Chootong P, Han ET. Immunogenicity of the Plasmodium vivax merozoite surface protein 1 paralog in the induction of naturally acquired antibody and memory B cell responses. Malar J 2017; 16: 354.

29. Cheng Y, Wang Y, Ito D, Kong DH, Ha KS, Chen JH, Lu F, Li J,
Wang B, Takashima E, Sattabongkot J, Tsuboi T, Han ET. The Plasmodium vivax merozoite surface protein 1 paralog is a novel erythrocyte-binding ligand of P. vivax. Infect Immun 2013; 81: 1585-1595.

30. Ahmed MA, Chu KB, Quan FS. The Plasmodium knowlesi Pk41 surface protein diversity, natural selection, sub population and geographical clustering: a 6-cysteine protein family member. PeerJ 2018; 6: e6141. 